\title{
An Ultra Low-Loss Silicon-Micromachined Waveguide Filter for D-Band Telecommunication Applications
}

\author{
James Campion*, Oleksandr Glubokov*, Adrían Gomez*, Aleksandr Krivovitca*, \\ Umer Shah*, Lars Bolander ${ }^{\dagger}$, Yinggang $\mathrm{Li}^{\dagger}$ and Joachim Oberhammer* \\ * Dept. of Micro and Nanosystems, KTH Royal Institute of Technology, Stockholm, Sweden. \\ jcampion@kth.se \\ $\dagger$ Ericsson Research, SE-417 56, Göteborg, Sweden
}

\begin{abstract}
A very low-loss micromachined waveguide bandpass filter for use in D-band $(110-170 \mathrm{GHz})$ telecommunication applications is presented. The $134-146 \mathrm{GHz}$ filter is implemented in a silicon micromachined technology which utilises a double H-plane split, resulting in significantly lower insertion loss than conventional micromachined waveguide devices. Custom splitblocks are designed and implemented to interface with the micromachined component. Compact micromachined E-plane bends connect the split-blocks and DUT. The measured insertion loss per unit length of the waveguide technology $(0.008$ $0.016 \mathrm{~dB} / \mathrm{mm}$ ) is the lowest reported to date for any micromachined waveguide at D-band. The fabricated 6-pole filter, with a bandwidth of $11.8 \mathrm{GHz}(8.4 \%)$, has a minimum insertion loss of $0.41 \mathrm{~dB}$, averaging $0.5 \mathrm{~dB}$ across its $1 \mathrm{~dB}$ bandwidth, making it the lowest-loss D-band filter reported to date in any technology. Its return loss is better than $20 \mathrm{~dB}$ across $85 \%$ of the same bandwidth. The unloaded quality factor of a single cavity resonator implemented in this technology is estimated to be 1600 .
\end{abstract}

Index Terms-D-band, bandpass filter, micromachined waveguide, waveguide filter, wireless link, telecommunication

\section{INTRODUCTION}

The continued growth of wireless data traffic, which has increased by a factor of 18 over the past 5 years [1], has pushed current wireless networks to the limit of their capacity. The increase in capacity required for future wireless communications can only be supported via an increase in system bandwidth. This factor, coupled with the expense and difficulty of constructing optical-fiber based networks, has created interest in wireless networks operating at frequencies above $100 \mathrm{GHz}$, where large swathes of continuous bandwidth are available. Such systems are enabled by developments in semiconductor design and processing technology. Modern low-cost, commercial semiconductor technologies, such as silicon germanium $\mathrm{BiCMOS}$, enable the large-scale implementation of transceiver circuits above $100 \mathrm{GHz}$. The band of frequencies between 110-170 GHz, known as the D-band, offers relatively low atmospheric attenuation $(<1 \mathrm{~dB} / \mathrm{km},[2])$, making it attractive for use in short/medium distance applications such as wireless backhaul. Theoretical link budget calcuations [2] have shown that wireless links operating at D-band can support data rates of up to $10 \mathrm{Gbps} / \mathrm{GHz}$. Practical system demonstrations report data rates of up to $48 \mathrm{Gbps}$ [3], confirming the potential of wireless links operating in this band.

A major limiting factor in the design of such systems is the low quality factor $(Q)$ of transmission lines implemented in these technologies, which prevents on-chip integration of lowloss passive components. High- $Q$ bandpass filters are required for channel selection in practical wireless links. As a result, all necessary filtering is typically performed off-chip in a separate transmission medium. In [4], channel selection is performed using a waveguide bandpass filter, implemented in a CNC milled metallic split-block technology. This 7-pole filter achieved an insertion loss of between $1-2.5 \mathrm{~dB}$ with a bandwidth of $11.8 \mathrm{GHz}(8.4 \%)$ centred at $139 \mathrm{GHz}$ [5]. Metallic split-block components are expensive, bulky and offer limited tolerances and feature sizes.

In this work, we report on the development of a siliconmicromachined waveguide bandpass filter for use in a Dband telecommunication system. Micromachining (MEMS) offers many benefits for the creation of high- $Q$, low-cost waveguide components. It allows for the use of complex geometries with small, accurate features and reduces fabrication costs by taking advantage of batch processing techniques. RFMEMS devices enable novel reconfigurable circuitry which cannot be implemented in other technologies [6]. Micromachined D-band filters by Zhao et al. [7] demonstrate low insertion loss $(0.45 \mathrm{~dB})$ but were measured with an un-calibrated system, resulting in a large uncertainty in the reported values.

\section{Filter Design}

The proposed waveguide filter consists of 6 cavities, directly coupled via inductive irises [8]. The dimensions of the filter and its coupling co-efficients are outlined in Fig. 1a. The filter was designed for a centre frequency of $144.75 \mathrm{GHz}$, which has been allocated for use in fixed wireless links, with a $3 \mathrm{~dB}$ bandwidth of $7.5 \mathrm{GHz}(5.2 \%)$. The simulated insertion loss of the filter is $0.26-0.8 \mathrm{~dB}$, while the return loss is greater than $23 \mathrm{~dB}$ in the passband (Fig. 1b). A conductivity of $2.1 \times 10^{7} \mathrm{~S} / \mathrm{m}$ was used during simulation to account for surface roughness effects.

Due to the design of the radio system, the proposed waveguide filter must be implemented such that its ports lie in the same plane. This required the design of custom metal split-blocks for test and verification purposes. The split-blocks are made of high-conductivity aluminium, realised in an E-plane split design using CNC milling. The finalised micromachined filters are inserted between a pair of split-blocks, which interface with standard WR-6 waveguides (Fig. 2a). Micromachined Eplane bends were used to transition between the split-blocks 


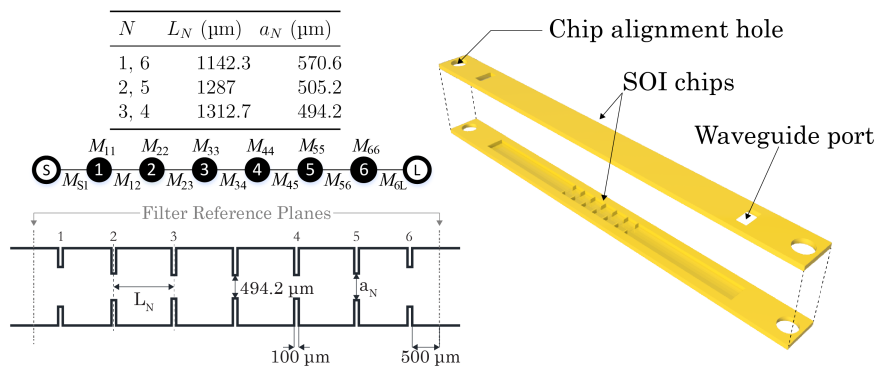

(a)

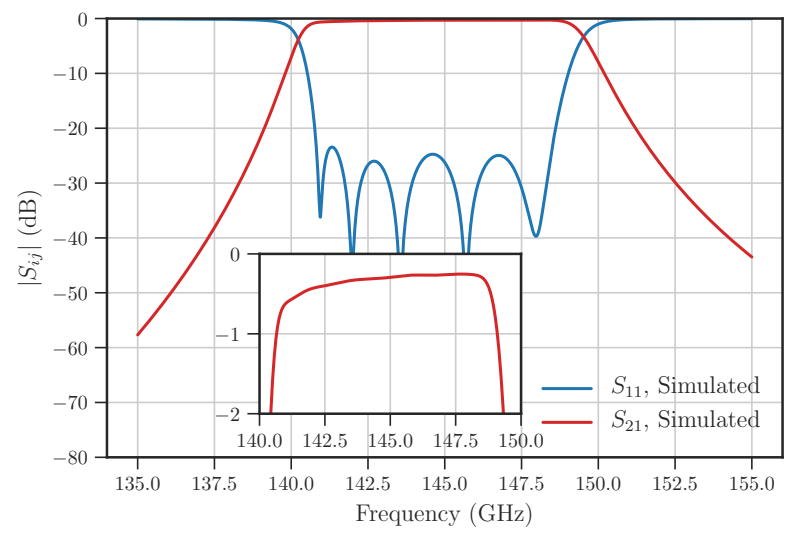

(b)

Fig. 1: (a) CAD model, cavity dimensions and coupling coefficients of the filter. $M_{S 1}=1.002, M_{12}=M_{56}=0.843$, $M_{23}=M_{45}=0.611, M_{34}=0.583, M_{i i}=0, i=1$ to 6 . (b) Simulated $S$-parameters of the filter, $\sigma=2.1 \times 10^{7} \mathrm{~S} \mathrm{~m}^{-1}$.

and the DUT. The simulated $S$-parameters of a single bend are shown in Fig. 4. The simulated transition has an insertion loss below $0.1 \mathrm{~dB}$ and return loss greater than $16 \mathrm{~dB}$ across the D-band.

\section{FABRicAtion AND ASSEMbly}

The filter was implemented in a low-loss silicon micromachined waveguide technology which utilises a double H-plane split [9]. The insertion loss of this technology is significantly lower than other micromachined waveguide implementations. Two separate silicon-on-insulator (SOI) wafers are used to realise the complete waveguides, which are split along their $\mathrm{H}$-plane. The height of each waveguide half is defined by the thickness of the SOI wafer's handle layer, allowing for precise control of this parameter.

Fabrication is performed in a three mask process, where photolithography is used to pattern the silicon oxide hard masks. The features of the filter are patterned in the handle layer of a single wafer via deep reactive ion etching. Discrete waveguide pieces are created by etching through the device and buried oxide layers of the SOI. Sputter deposition is used to apply $1 \mu \mathrm{m}$ of gold to the chips. The individual waveguide halves are then assembled prior to thermo-compression bonding at $250{ }^{\circ} \mathrm{C}$. Alignment between the waveguide halves is facilitated

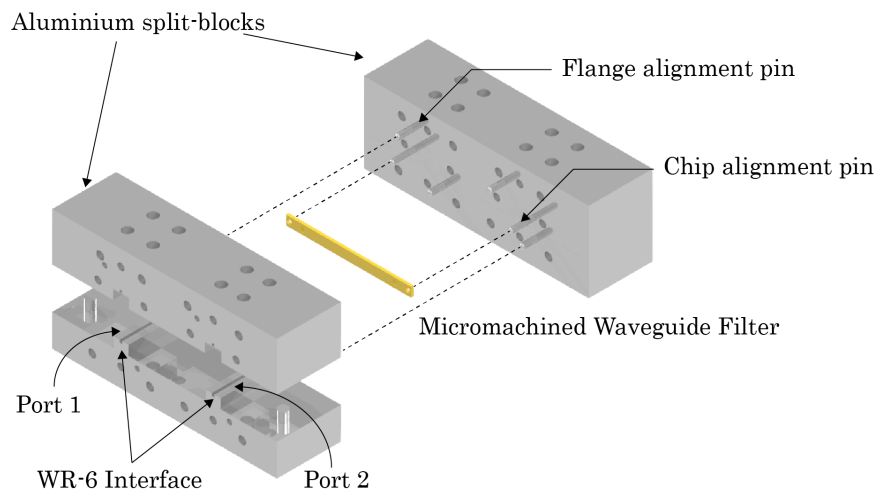

(a)

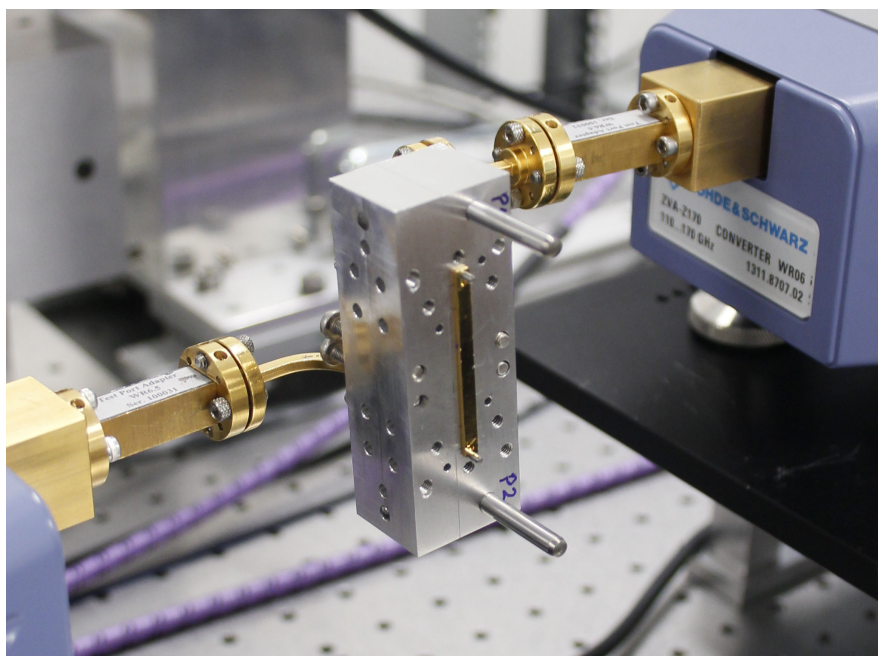

(b)

Fig. 2: (a) CAD model of the metallic split-block assembly. (b) A fabricated micromachined filter, mounted on the split-block assembly.

via self-alignment features patterned in the handle layer of each piece. The E-plane bends are realised by offsetting the etched area in the bottom chip, creating a waveguide step. An example of an assembled chip is shown in Fig. $2 b$.

\section{RF CHARACTERISATION}

The RF performance of the filters was measured using Rohde \& Schwarz ZVA-Z170 frequency extenders driven by a ZVA-24 VNA. The filter was mounted between the split-blocks detailed in Section II, which were connected to the frequency extenders via H-plane waveguide bends (Fig. 2b). Elliptical alignment holes [10] were implemented on all micromachined components to facilitate highly-accurate, repeatable alignment to the metallic split-blocks. Calibration was performed using an integrated LRL calibration kit, where the Thru was a nonzero-length straight waveguide and the Line was implemented as a $\lambda / 4$ dog-leg. Following determination of the complex propagation constant of the waveguide the reference planes were shifted to the filter ports (Fig. 1a). This information 


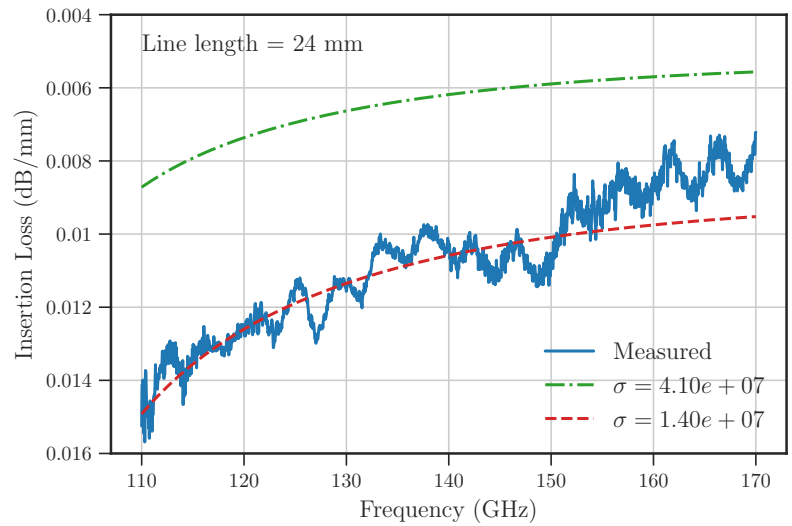

Fig. 3: Measured insertion loss per unit length of the micromachined waveguide, compared to theoretical values for conductivities $\sigma=4.1 \times 10^{7} \mathrm{~S} \mathrm{~m}^{-1}, \sigma=1.4 \times 10^{7} \mathrm{~S} \mathrm{~m}^{-1}$

also allows for the insertion loss of the waveguide technology to be determined. The resulting insertion loss per unit length is between $0.008-0.016 \mathrm{~dB} / \mathrm{mm}$ across the Dband (Fig. 3). This corresponds to the theoretical conductive loss of an ideal rectangular waveguide with a conductivity of $\sigma=1.4 \times 10^{7} \mathrm{~S} \mathrm{~m}^{-1}$ [11]. This effective conductivity value inherently includes the effects of the waveguide's surface roughness. To the best of the authors' knowledge, this represents the lowest-loss D-band silicon micromachined waveguide published to date. From the measured data, a single cavity resonator implemented in this technology is expected to have an unloaded $Q$ factor of $\sim 1600$.

The performance of the E-plane bend transition was verified via a two-tier, one-port calibration routine [12], calibrated firstly at the split-block faces and then at the reference plane inside the micromachined waveguide, where 5 offsetshorts were used as standards. Two-port $S$-parameters of the transition were extracted via de-embedding of the resulting error networks. Its insertion loss is below $0.5 \mathrm{~dB}$ and return loss greater than $15 \mathrm{~dB}$ across the entire band (Fig. 4).

The measured $S$-parameters of the bandpass filter are plotted in Fig. 5. The filter has a minimum insertion loss of $0.41 \mathrm{~dB}$, averaging $0.5 \mathrm{~dB}$ across its $1 \mathrm{~dB}$ passband, which represents, to the best of the authors' knowledge, the lowest insertion loss reported for a D-band filter of similar order and bandwidth in any technology to date. The passband return loss is greater than $20 \mathrm{~dB}$ across $85 \%$ of its $1 \mathrm{~dB}$ bandwidth. The fabricated filter exhibits a decrease in a centre frequency by $4.75 \mathrm{GHz}$. This was attributed to underetching of the waveguide sidewalls during fabrication, which increased the size of all 6 cavities and changed iris width/thickness, thereby detuning the filter.

\section{CONClusion}

A very low-loss micromachined waveguide filter for use in D-band telecommunications systems has been presented. The filter is implemented in an H-plane split technology, where each waveguide half comprises a single SOI wafer.

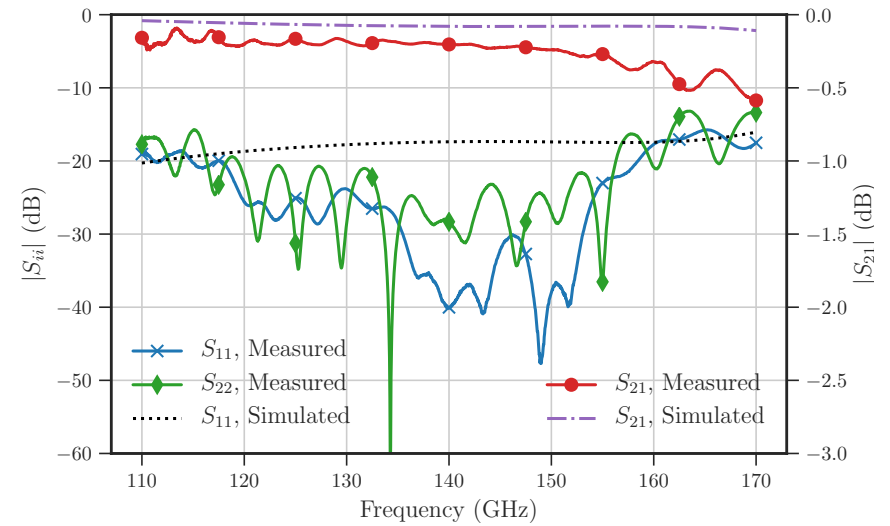

Fig. 4: Measured $S$-parameters of the micromachined E-plane bend transition.

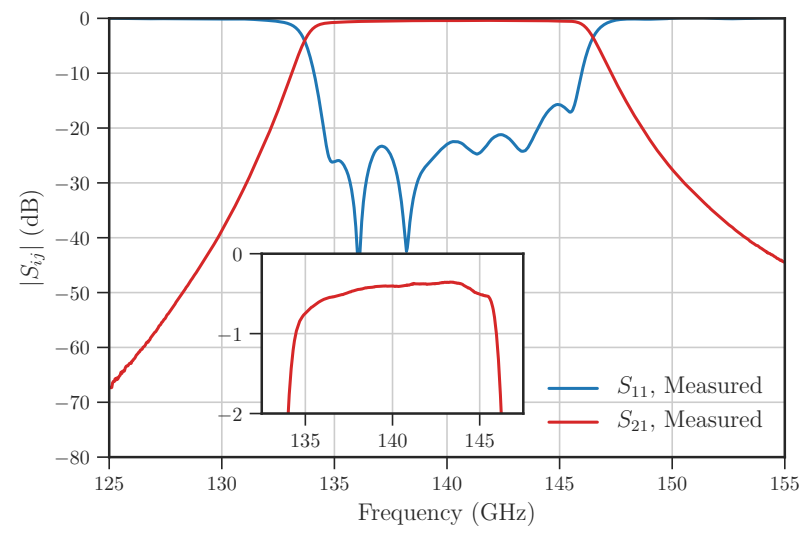

Fig. 5: Measured $S$-parameters of the micromachined waveguide filter.

Custom metallic split-blocks were designed and fabricated to connect the micromachined filter to standard WR-6 interfaces. The proposed filter achieves an insertion loss of $0.41 \mathrm{~dB}$ at $143 \mathrm{GHz}$, with an average of $0.5 \mathrm{~dB}$ across its passband. As such, the filter presented here has the lowest insertion loss of any D-band filter yet reported. This performance is due to the low insertion loss of the waveguide technology itself which, ranging between $0.008-0.016 \mathrm{~dB} / \mathrm{mm}$, is the lowest of any Dband waveguide reported to date. This performance makes micromachined waveguide components extremely promising for use in future telecommunication applications above $100 \mathrm{GHz}$.

\section{ACKNOWLEDGEMENT}

This work has received funding from the Swedish Foundation for Strategic Research Synergy Grant Electronics SE13007, the European Research Council (ERC) under the European Union's Horizon 2020 research and innovation programme (grant agreement No 616846) and the European Union's Horizon 2020 research and innovation programme under grant agreement No 644039 (M3TERA). This research 
made use of scikit-rf, an open-source Python package for RF and Microwave applications.

\section{REFERENCES}

[1] "Cisco Visual Networking Index: Global Mobile Data Traffic Forecast Update, 2016-2021 White Paper ," Tech. Rep.

[2] T. Schneider, A. Wiatrek, S. Preußler, M. Grigat, and R.-P. Braun, "Link Budget Analysis for Terahertz Fixed Wireless Links," IEEE Transactions on Terahertz Science and Technology, vol. 2, no. 2, pp. 250-256, 2012.

[3] S. Carpenter, D. Nopchinda, M. Abbasi, Z. S. He, M. Bao, T. Eriksson, and H. Zirath, "A D-Band 48-Gbit/s 64-QAM/QPSK Direct-Conversion I/Q Transceiver Chipset," IEEE Transactions on Microwave Theory and Techniques, vol. 64, no. 4, pp. 1285-1296, 2016.

[4] C. Wang, C. Lin, Q. Chen, B. Lu, X. Deng, and J. Zhang, "A 10-Gbit/s Wireless Communication Link Using 16-QAM Modulation in 140GHz Band," IEEE Transactions on Microwave Theory and Techniques, vol. 61, no. 7, pp. 2737-2746, July 2013.

[5] W. Cheng, L. Bin, L. Jie, and D. Xianjin, "140GHz Waveguide H ladder Bandpass Filter," in Microwave and Millimeter Wave Technology (ICMMT), 2012 International Conference on, vol. 2. IEEE, 2012, pp. $1-4$.

[6] U. Shah, T. Reck, H. Frid, C. Jung-Kubiak, G. Chattopadhyay, I. Mehdi, and J. Oberhammer, "A 500 - 750 GHz RF MEMS Waveguide Switch," IEEE Transactions on Terahertz Science and Technology, vol. 7, no. 3, pp. 326-334, May 2017.

[7] X. H. Zhao, J. F. Bao, G. C. Shan, Y. J. Du, Y. B. Zheng, Y. Wen, and C. H. Shek, "D-band Micromachined Silicon Rectangular Waveguide Filter," IEEE Microwave and Wireless Components Letters, vol. 22, no. 5, pp. 230-232, 2012.

[8] G. L. Matthaei, . Young, Leo, and E. M. T. Jones, Microwave Filters, Impedance-Matching Networks, and Coupling Structures. Dedham, Mass. : Artech House, 1980, reprint. Originally published: New York : McGraw-Hill, 1964.

[9] B. Beuerle, J. Campion, U. Shah, and J. Oberhammer, "A Very Low Loss 220-325 GHz Silicon Micromachined Waveguide Technology," IEEE Transactions on Terahertz Science and Technology, pp. 1-3, 2018.

[10] J. Campion, U. Shah, and J. Oberhammer, "Elliptical Alignment Holes Enabling Accurate Direct Assembly of Micro-chips to Standard Waveguide Flanges at sub-THz Frequencies," in 2017 IEEE MTT-S International Microwave Symposium (IMS), June 2017, pp. 1262-1265.

[11] E. Hammerstad and O. Jensen, "Accurate Models for Microstrip Computer-Aided Design," in Microwave Symposium Digest, 1980 IEEE MTT-S International. IEEE, 1980, pp. 407-409.

[12] T. J. Reck, L. Chen, C. Zhang, A. Arsenovic, C. Groppi, A. Lichtenberger, R. M. Weikle, and N. S. Barker, "Micromachined Probes for Submillimeter-Wave On-Wafer Measurements-Part II: RF Design and Characterization," IEEE Transactions on Terahertz Science and Technology, vol. 1, no. 2, pp. 357-363, 2011. 